\title{
Arbolado Urbano y Confort Térmico en el Hábitat Pozarricense
}

\section{Urban Trees and Thermal Comfort in the Pozarricense Habitat}

\author{
Jesús Martínez Bocardiª-Blanca Inés Aguilar Frías ${ }^{b}$ \\ a Observatorio Urbano y de Arquitectura Sustentable, Facultad de Arquitectura, \\ Universidad Veracruzana, Poza Rica, México. \\ Contacto: jmartinezb@uv.mx \\ b Responsable del Observatorio Urbano y de Arquitectura Sustentable, \\ Facultad de Arquitectura, Universidad Veracruzana, Poza Rica, México. \\ Contacto: baguilar@uv.mx
}

Recibido: 19 de agosto de 2020

Aceptado: 15 de octubre

RESUMEN: La ciudad de Poza Rica de Hidalgo, ubicada en la zona norte del estado de Veracruz; debe su gran desarrollo a que fue una de las ciudades petroleras más importantes en los años setenta gracias a su posición geográfica, lo que le permitió ser el núcleo urbano para los municipios aledaños. Debido a su constante tránsito vehicular y peatonal, la localidad ha llegado a sufrir de congestiones viales en el Boulevard Ruiz Cortines, Lázaro Cárdenas y la Avenida Central Poniente. Se implementaron estrategias en zonas específicas con la finalidad de mejorar la circulación vial en las horas de mayor tránsito; un ejemplo es el distribuidor vial construido en el periodo 2012-2015. Para su construcción se consideraron 3.2 kilómetros que pasan por la zona centro y se derribaron más de 200 árboles, de los cuales, muchos se consideraban en peligro de extinción sin pensar en cómo afectaría esto a largo plazo. Como consecuencia las temperaturas aumentaron en la zona centro, ya que cuenta con una mínima cantidad de árboles y en su mayoría se encuentran en mal estado. La valorización de un árbol se encuentra en segundo término al momento planificar las vialidades. Debido a esto, se pretende realizar una catalogación de las especies, con la finalidad de conocer qué tipo de flora se desarrolla mejor de acuerdo con las condiciones climatológicas y topográficas. Así, se tomará en cuenta que arboles deben reubicarse, retirarse o replantar, para una mejor conservación de estas especies. Esta estrategia beneficiará para mejorar la calidad del aire, en el embellecimiento de la imagen urbana, en la reducción de temperatura y en eficientar el 
presupuesto que se destina a estas zonas. Con lo anterior se generará un documento de consulta abierta para la planificación de áreas verdes, parques y jardines, diseño de espacios públicos con la finalidad de mitigar las altas temperaturas en la isla de calor identificada dentro de la ciudad.

Palabras clave: Confort térmico; Isla de calor; Climatología; Planificación; Imagen urbana.

ABSTRACT: The city of Poza Rica de Hidalgo, located in the northern zone of the state of Veracruz; owes its great development to that it was one of the most important oil cities of the second half of the s. XX, and its geographical position, which allowed it to be the main urban nucleus for the surrounding municipalities. Due to its constant vehicular traffic, the town has suffered from traffic congestions in Ruiz Cortines Boulevard, Lázaro Cárdenas, and Central Poniente Avenue. Strategies were implemented in specific areas with the purpose of improving road circulation during the busiest hours; an example is the road distributor built in the period 2012-2015. For its construction, 3.2 kilometers were considered to pass through the central zone and more than 200 trees were felled, of which many were considered in danger of extinction without thinking about how this would affect in the long term. As a result, temperatures increased in the central area, since it has a minimum number of trees, most of which are in poor condition, to reduce exposure to solar rays from the surface. The valuation of a tree is in second term when planning the roads. Due to this, it is intended to analyze the current status of the green area of the Boulevard Adolfo Ruiz Cortines and determine which trees should be removed or relocated, according to the species that best adapt to the climatic and topographic conditions, for a better conservation of the individuals - This strategy seeks to contribute to the improvement of air quality, the reduction of temperature, the embellishment of the urban image, and the decrease in the budget allocated to this area. With the above, an open consultation document will be generated for the planning of green areas, parks and gardens, and design of public spaces, to mitigate the high temperatures in the heat islands identified within the city.

Keywords: Thermal comfort, Heat island, Climatology, Planning, Urban image.

\section{Introducción}

1

n ocasiones el crecimiento de las ciudades produce espacios remanentes ocupados por la naturaleza nativa, que pasan a formar parte de las áreas verdes urbanas; aunque los actuales enfoques sustentables de desarrollo implican un diseño y una gestión de estos espacios que favorezcan la integración armónica de la urbe con su entorno; con el apoyo de 
disciplinas como la planificación, la gestión ambiental, el diseño urbano y la arquitectura de paisaje. Los parques urbanos juegan un papel importante para la población, cumpliendo una doble función: por un lado contribuyen en el aspecto social y cultural, siendo espacios públicos, de recreación, ocio y democratizadores del espacio (Sierra y Ramírez, 2010); además de su importante aportación al entorno ecológico, mediante una variedad de funciones como la generación de oxígeno, la regeneración de mantos freáticos, la regulación del micro clima, y como barreras de amortiguación de polvo y ruido en la ciudad. Aunado a esto, la presencia de espacios verdes representa una mejora en la imagen y valoración de la zona en que se ubican.

\section{Calidad de vida y medio ambiente}

El medio ambiente es un fenómeno complejo, y la manera en que se relaciona con la salud y la calidad de vida de las personas constituye un elemento de creciente interés.

Respecto a la relación entre salud y medio ambiente, ciertos factores crean condiciones en las que se pueden desarrollar distintas afectaciones ambientales para la salud. Por un lado, está el factor demográfico, que multiplica el impacto de la actividad humana, particularmente en el consuno de recursos y la producción de residuos; por otro lado se encuentra el fenómeno urbano, y la depredación del medio natural resultante, ante la ausencia o ineficacia de políticas sustentables; además de otros factores como la pobreza y la inequidad social, que influyen en el estado del medio ambiente en que viven las personas (Baldi y García, 2005). Tanto el entorno en que se vive, como la salud pueden ser componentes de la percepción de la calidad de vida; y contribuyen a su vez al desarrollo humano y social.

\subsection{Derecho a la ciudad y al desarrollo humano.}

El modo en que contribuyen los espacios verdes de la ciudad al objetivo de alcanzar un desarrollo urbano sustentable puede abordarse mediante un enfoque ecológico, en donde la presencia de estos espacios permite conservar y mejorar las condiciones del sistema ecológico urbano, igualmente con un enfoque relacionado a la satisfacción de un derecho. La población urbana tiene derechos reconocidos por distintas instancias, desde locales hasta internacionales, tendientes a garantizar su desarrollo humano integral, como habitantes de la ciudad (Ciudades, 2011).

Las personas que habitan en la ciudad tienen derecho a una ciudad constituida como comunidad política municipal que asegure condiciones adecuadas de vida y procure la 
convivencia entre todos sus habitantes; todas las personas que habitan en la ciudad tienen derecho a disponer de espacios y recursos para la práctica de una ciudadanía activa. En la Carta-agenda mundial de derechos humanos en la ciudad se expresa que todas las personas que habitan en la ciudad tienen derecho a un desarrollo urbano de calidad, respetuoso con el medio ambiente; que la ciudad tome las medidas necesarias para obtener un medio ambiente urbano sano, procurando en particular mejorar constantemente la calidad del aire; asimismo, que en el ejercicio de su responsabilidad, los habitantes de la ciudad actúan de manera compatible con el respeto a la preservación del medio ambiente, el ahorro energético y el buen uso de los equipamientos públicos. Es en este sentido que las áreas verdes del boulevard resultan importantes para la consecución de estos objetivos.

\subsection{Sustentabilidad y servicios ambientales}

Un referente indispensable de la sustentabilidad es la capacidad de carga, concepto que se refiere a qué tanto puede la naturaleza soportar la actividad humana sin resultar afectada de manera permanente. Los recursos naturales son finitos y algunos no son renovables, por lo que tienen un límite de tolerancia a su explotación y a las actividades humanas. En un municipio, la capacidad de carga es el consumo de los recursos naturales que el territorio puede sostener sin desequilibrar el funcionamiento y la productividad de los ecosistemas (SEDESOL, 2012). Los espacios verdes de las ciudades representan el vínculo de la urbe con su entorno, y juegan diversos papeles con diferentes niveles de impacto. En este sentido, los servicios ambientales son aquellos servicios que brinda el medio ambiente, que tienen un enorme valor para la vida del hombre, aunque muchas veces no son cuantificados en términos económicos. Entre estos servicios se encuentran la producción de aire limpio, de agua, conservación de la biodiversidad, el paisaje, entre otros.

\section{Isla de calor y vegetación urbana}

Un entorno más equilibrado favorece a las condiciones del desarrollo humano; pero cuando uno de los componentes del sistema se altera, puede producir afectaciones que dependen de la magnitud y frecuencia de las desviaciones de los indicadores. Uno de los aspectos que caracterizan el ambiente urbano es la elevada temperatura predominante. El clima urbano es el resultado del efecto de la radiación solar sobre la superficie urbana, que se transfiere a su 
vez de vuelta a la atmósfera. Las ciudades se componen de un conjunto de edificaciones en las que sus materiales conforman una superficie que refleja gran parte de la radiación solar, en lugar de absorberla o dispersarla, como sucede con la superficie natural. La capa vegetal brinda protección a la superficie mediante diferentes estratos de árboles y arbustos, a la vez que favorece la evapotranspiración, que interviene en el balance calorífico (Barradas, 2013).

La escasez de áreas vegetales combinada con materiales de construcción de alta conductividad térmica favorece el calentamiento del aire cercano a la superficie del suelo, generando el fenómeno de la isla de calor urbana (ICU), que se caracteriza principalmente porque la temperatura del aire es más elevada en el área urbana que en sus alrededores. Por otra parte, los procesos de combustión derivados de la industria y el transporte, además de la contaminación, incrementan el efecto de calentamiento, que no puede dispersarse en la atmósfera debido a la aglomeración de estructuras elevadas que dificulta la ventilación en las ciudades.

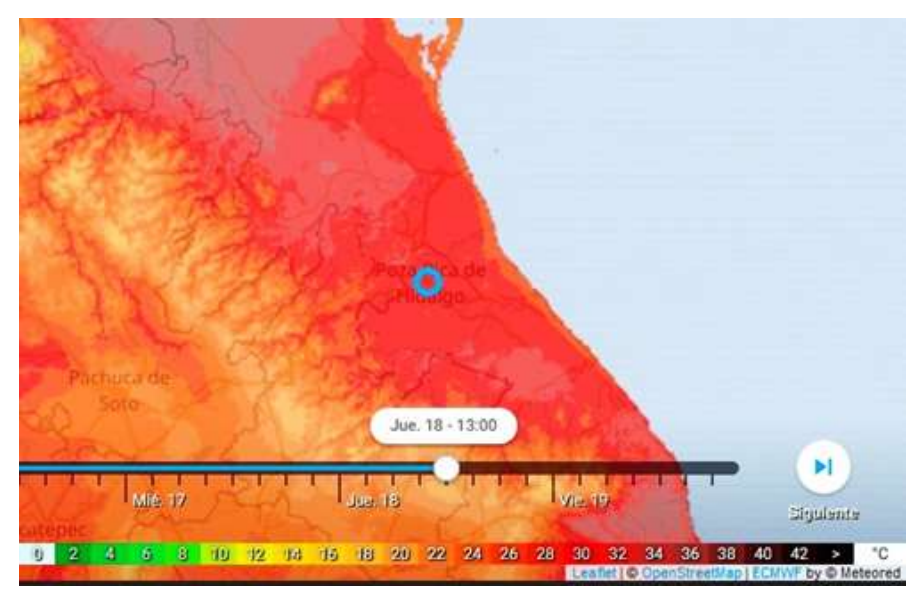

Figura 1: Mapa de calor del norte de Veracruz, donde se ubica la ciudad de Poza Rica, con temperaturas promedio de $24.4^{\circ} \mathrm{C}$. Fuente: Meteored

El aumento en la temperatura urbana tiene un efecto directo en el índice de confort térmico humano, causando en la población desde incomodidad hasta estrés por calor, además de afectaciones a la salud; lo cual repercute a su vez al incremento del consumo de energía para refrescar el interior de las edificaciones. En este sentido, la vegetación urbana desempeña un papel importante en el microclima de las ciudades; las áreas verdes tienen la capacidad de absorber energía radiante a través de la evaporación y la transpiración, como resultado, su entorno es más fresco y húmedo que otros lugares. 


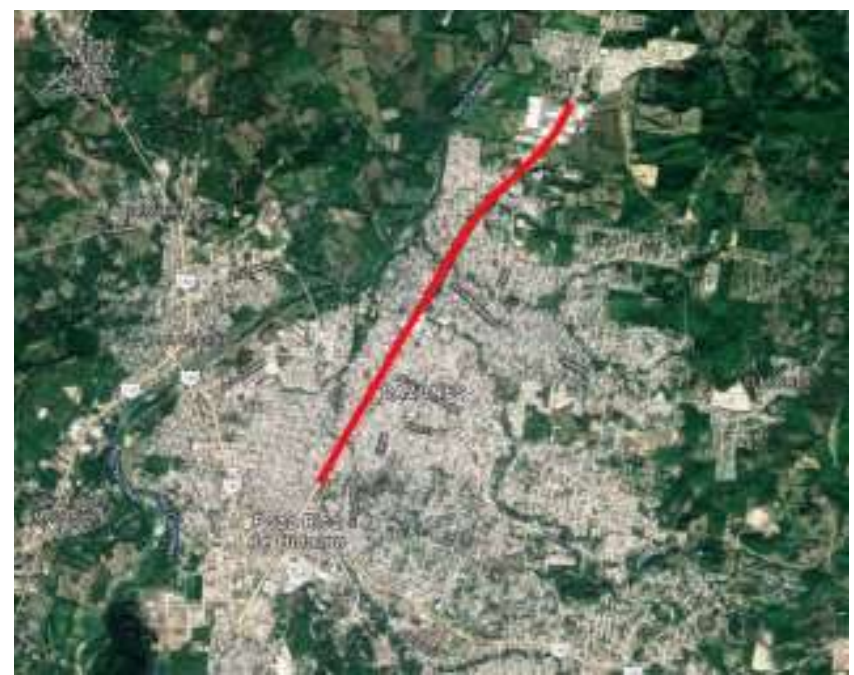

Figura 2: Ubicación del bulevar Adolfo Ruiz Cortines en la traza urbana de Poza Rica. Fuente: Google Maps

\subsection{Clima urbano y población}

Si las diferencias de temperatura urbana y de su entorno -o rural- manifiestan el calentamiento relativo de la ciudad debido al proceso de isla de calor urbana, el factor determinante para que esto ocurra es la transformación del medio por el ser humano, o antropización (Kurbán y Grasso, 2016).

Las acciones de la antropización en las ciudades resultan en desbalances o alteraciones de diversos sistemas que se presentan sencillamente en el entorno natural, como son principalmente: el ciclo de radiación solar, al resultar devastada la cubierta vegetal; el ciclo del agua, alterando los procesos de evaporación; el flujo natural del aire, construyendo barreras o estructuras; todo ello potenciado por la emisión de contaminantes atmosféricos, que incrementan el efecto invernadero. Este conjunto de acciones repercute en la modificación del clima urbano, y finalmente en la calidad de vida de los habitantes.

\section{Caso de estudio}

Poza Rica cuenta con dos ejes principales de comunicación, en sentido norte-sur está el boulevard Adolfo Ruiz Cortines, que con una longitud de más de 5,300 mts. es la avenida más larga y amplia de la ciudad, y cuenta con un camellón ocupado en su mayor parte por áreas verdes. La temperatura promedio es de $24.4^{\circ} \mathrm{C}$, la precipitación pluvial media anual es de 1,010 
mm. El ecosistema predominante en el municipio es el de bosque mediano perennifolio con especies de guarambo, jonote, guanactle y sangrado.

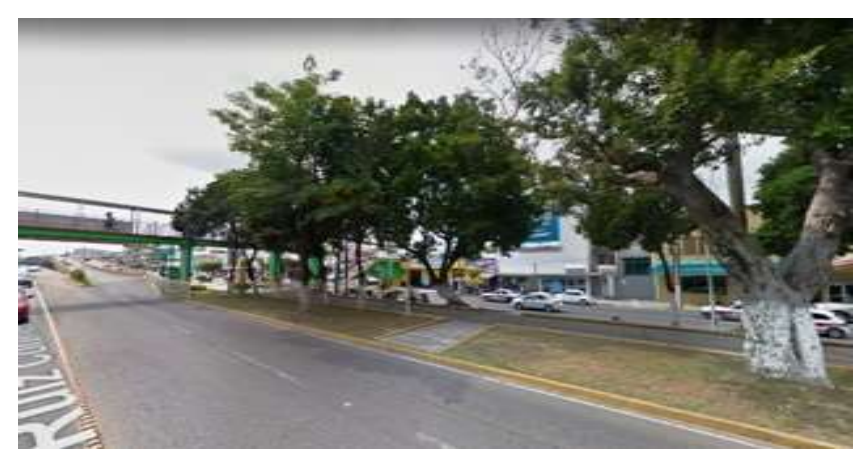

Figura 3: Extremo sur del bulevar Adolfo Ruiz Cortines, donde se observa su interrupción en la vegetación por el distribuidor vial, al fondo. Fuente: Google

El bulevar Adolfo Ruiz Cortines ha sido objeto de distintas modificaciones, entre las principales se encuentran: la reducción del camellón central de casi 2 mts. a cada lado entre las colonias Obrera y Manuel Ávila Camacho para agilizar la circulación vehicular, eliminando las banquetas laterales del camellón; la construcción del distribuidor vial, que en el tramo que comprende las colonias Obrera y Obras Sociales representó la remoción de alrededor de 200 árboles, algunos de los cuales se consideran en peligro de extinción (Figura 3); y la extensión del camellón desde la colonia La Rueda hasta la colonia Primavera (Figura 4).

También se han realizado diferentes trabajos de remodelación de andadores y equipamiento, así como modificación del área verde, principalmente con fines decorativos y de imagen urbana. En estos casos los especímenes arbóreos utilizados incluyen palmas, lirios persas y crotos, entre otros, que requieren riego constante para su preservación, lo que resulta inadecuado para el clima predominante de la ciudad, y la escasez de agua potable que padecen amplios sectores de la población. 


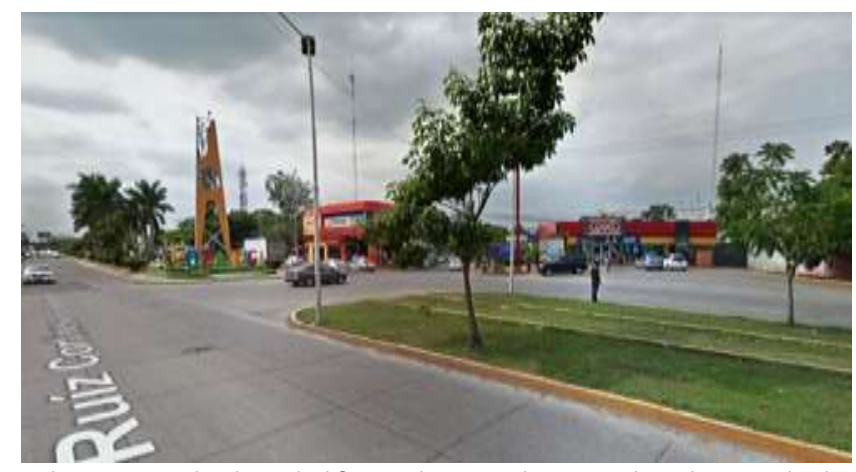

Figura 4: Blvd. Adolfo Ruiz Cortines, a la altura de la col. La Rueda, donde inicia su más reciente ampliación, en primer plano. Fuente: Google

Una de las más recientes acciones consistió en reforestar el extremo norte del camellón sembrando 37 árboles, entre los que destacan palo de rosa, del sueño y melinas (Figuras 5 y 6). Esta iniciativa resultó más acertada por el tipo de especies seleccionadas, como el palo de rosa (tabebuia rosea) que es originario de las regiones tropicales de América y su distribución geográfica abarca desde México, Centroamérica, y El Caribe, de tipo caducifolio, alcanza alturas hasta de $30 \mathrm{~m}$. y un diámetro hasta de $1 \mathrm{~m}$., de copa estratificada y abierta (Niembro et al., 2010). En los diferentes espacios que conforman el camellón del boulevard se encuentran árboles que tienen distintas características, como son: especie, edad y condición (o salud arbórea); cada uno de ellos se adapta en mayor o menor medida a las condiciones del medio en el que predomina un clima caluroso con un prolongado estiaje, lo que vuelve costoso -económica y socialmente- el riego continuo que necesitan las especies no adaptadas. Algunos árboles de edad avanzada presentan condiciones de deterioro, y, por otro lado, los árboles jóvenes recientemente plantados pueden ser deteriorados si no se tiene el cuidado necesario.

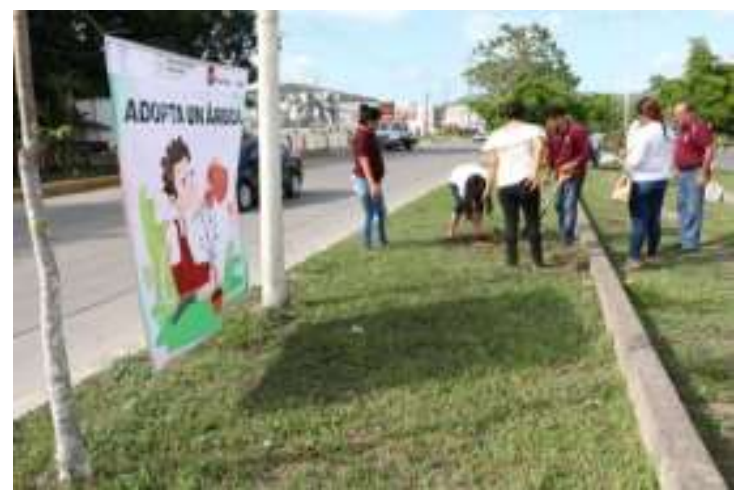

Figura 5: Reforestación del Blvd. Adolfo Ruiz Cortines, a la altura de la col. La Rueda, al norte de la ciudad. Fuente: Gobierno de Poza Rica. 2019. 


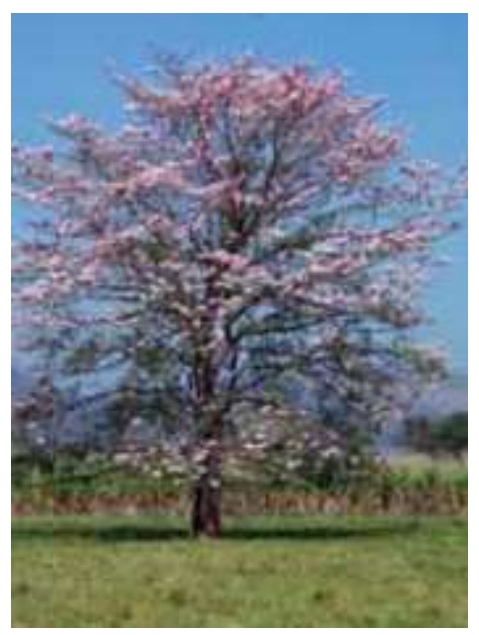

Figura 6: Tabebuia rosea o palo de rosa. Árbol originario de las regiones tropicales de América. Fuente: Niembro et al., 2010

También se encuentran numerosos arbustos, principalmente en la sección más antigua del camellón, que cumplen principalmente una función estética. Aunque varios de estos ejemplares no pertenecen a especies adecuadas para el clima de la región, por lo que se deterioran o se pierden. El extremo norte del camellón presenta ejemplares más jóvenes, y en número más reducido; además de que carece de arbustos, y solamente una cubierta de pasto protege el terreno de la radiación solar y la erosión pluvial y del viento; además carece de pavimento. En cuanto al equipamiento con el que cuenta el boulevard, éste se ubica principalmente en el camellón, y consta de andador central, espacios para sentarse, una plaza, una fuente, monumentos, y más recientemente, espacios para ejercitarse, y elementos escultóricos en el extremo sur (Figura 7). 


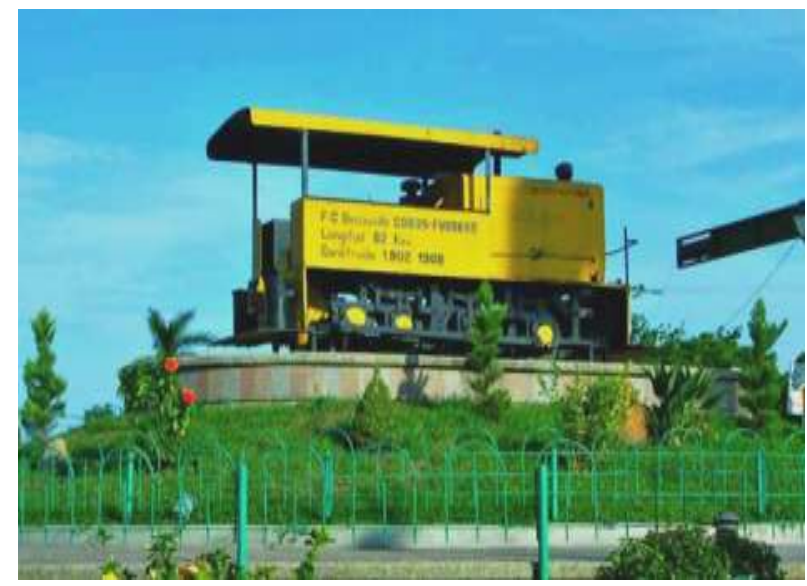

Figura 7: Monumento a La Maquinita, Poza Rica Corría en la antigua vía Cobos-Furbero, actual bulevar Ruiz Cortines. Fuente: Gobierno del Estado.

\subsection{Propuesta y recomendaciones}

A partir de un diagnóstico del estado actual de las áreas verdes del bulevar Adolfo Ruiz Cortines, se pretende identificar alternativas que contribuyan a mejorar el funcionamiento y el disfrute de éste importante espacio; y mediante un amplio proyecto urbano que incluya propuestas sustentables, se consiga al mismo tiempo la reducción del efecto isla de calor, todo ello sin dejar de lado el aspecto vial.

Una primera etapa consiste en abordar el tema de la flora para determinar, mediante el análisis de las distintas especies, los individuos arbóreos mejor adaptados a las condiciones climáticas y geográficas -nativos o no- para asegurar su supervivencia, y de esta manera reducir aspectos como el riego y el cuidado, manteniendo criterios de diseño del paisaje urbano. Otras vialidades, al igual que el bulevar Adolfo Ruiz Cortines, tienen un camellón central. La avenida 10, en la colonia Cazones, la avenida Uruguay en la colonia 27 de septiembre, y la avenida Manlio F. Altamirano en la colonia Tajín; con diferentes longitudes, cada una cuenta con camellones de distintas características, que a su vez cumplen diversas funciones dependiendo de sus características y de los usuarios que los frecuentan. Por lo que el estudio del bulevar Adolfo Ruiz Cortines puede servir de precedente para analizar esos espacios públicos y conocer su aportación al mejoramiento de su entorno. 


\section{Referencias}

Baldi, G., García, E. 2005. Calidad de Vida y Medio Ambiente. La psicología ambiental. Universidades, núm. 030. pp. 10, 12.

Barradas, V. 2013. La Isla de Calor Urbana. Oikos no. 7. pp. 17-20.

Ciudades y Gobiernos Locales Unidos. 2011. Carta-Agenda Mundial de Derechos Humanos en la Ciudad. Comisión de inclusión social, democrática participativa y derechos humanos. Florencia, Italia. pp. 13.

Kurbán, A. y Grasso C. 2016. Confort térmico en espacios verdes urbanos de ambientes áridos. Revista Hábitat Sustentable, no 1. pp. 35

Niembro, A., Vázquez, M., Sánchez, O. 2010. Árboles de Veracruz. 100 especies para la reforestación estratégica. Comisión Organizadora del Estado de Veracruz de Ignacio de la Llave para la Conmemoración del Bicentenario de la Independencia Nacional y del Centenario de la Revolución Mexicana. pp. 214, 215.

SEDESOL. 2012. Guía para el Desarrollo Local Sustentable. SEDESOL, Banco Mundial. México. pp. 20, 21.

Sierra, I., Ramírez, J. 2010. Los parques como elementos de sustentabilidad de las ciudades. Revista Fuente, 2(5) 6, 7pp.

El Heraldo de Poza Rica. 2016. Arrancan las acciones para mejorar la ciudad. Recuperado de http://elheraldodepozarica.com.mx/estado/poza-rica/37952-arrancan-las-accionespara-mejorar-la-ciudad.html.

Gobierno de Poza Rica. 2018. Boletín 298 del 13 de julio de 2018. Programa municipal de refprestación. Recuperado de https://www.gobiernodepozarica.gob.mx/boletin/continua-programa-municipal-dereforestacion-en-poza-rica-9b5

La jornada. 2017. Obra de rehabilitación del bulevar Ruiz Cortines en Poza Rica, atrasada 5 meses. Recuperado de http://www.jornadaveracruz.com.mx/Post.aspx?id=170509_071659_918 